\title{
Half-metal phases in a quantum wire with modulated spin-orbit interaction
}

\author{
D. C. Cabra, ${ }^{1}$ G. L. Rossini, ${ }^{1}$ A. Ferraz, ${ }^{2}$ G. I. Japaridze, ${ }^{3,4}$ and H. Johannesson ${ }^{5}$ \\ ${ }^{1}$ IFLP-CONICET, Departamento de Física, Universidad Nacional de La Plata, CC 671900 La Plata, Argentina \\ ${ }^{2}$ International Institute of Physics - UFRN, Department of Experimental and Theoretical Physics - UFRN, Natal, Brazil \\ ${ }^{3}$ Ilia State University, Faculty of Natural Sciences and Engineering, Cholokashvili avenue 3-5, 0162 Tbilisi Georgia \\ ${ }^{4}$ Andronikashvili Institute of Physics, Tamarashvili str. 6, 0177 Tbilisi, Georgia \\ ${ }^{5}$ Department of Physics, University of Gothenburg, SE 41296 Gothenburg, Sweden
}

(Received 25 August 2017; published 17 November 2017)

\begin{abstract}
We propose a spin filter device based on the interplay of a modulated spin-orbit interaction and a uniform external magnetic field acting on a quantum wire. Half-metal phases, where electrons with only a selected spin polarization exhibit ballistic conductance, can be tuned by varying the magnetic field. These half-metal phases are proven to be robust against electron-electron repulsive interactions. Our results arise from a combination of explicit band diagonalization, bosonization techniques, and extensive density matrix renormalization group computations.
\end{abstract}

DOI: 10.1103/PhysRevB.96.205135

\section{INTRODUCTION}

The ability to control and manipulate electron spins with an efficiency comparable to that of present-day (charge) electronics is one of the major goals of modern spintronics [1-3]. As it comes to applications, fabrication of a device providing spin-dependent currents is a central issue [4]. A seminal blueprint for a spin-filtering scheme was proposed in a paper published more than two decades ago [5]. The Datta-Das transistor uses a conductor contacted to a ferromagnetic source and drain, subject to a Rashba spin-orbit interaction (SOI) [6]. Depending on the spin orientations in the source and in the drain, the current flowing through the device can be controlled by rotating the spins of the incoming electrons, using a gate voltage to tune the strength of the SOI $[7,8]$. Progress notwithstanding [9], reliable injection of spin-polarized electrons from a ferromagnet into a semiconductor remains a challenge, and so does the very realization of a functional device producing spin-dependent currents.

Because a SOI couples spin and momentum of charge carriers, it also provides a differentiated effect on each spin polarization. This opens a window for a spin-filtering regime, where only electrons with one spin polarization carry current, while electrons with the opposite spin polarization are gapped. This possibility is most profoundly displayed in the case of one-dimensional conductors where a uniform Rashba SOI leads to a spin-dependent shift of the electron dispersion by a momentum $\tau q_{0}$, with $\tau=+,-$ the spin polarization along an axis determined by the SOI [10]. A Peierls-type mechanism for a spin-based current switch was identified in Ref. [11], where it was shown that a spatially smooth modulated Rashba SOI coupling opens both charge and spin gaps in the system at commensurate band fillings. Such an interaction could be generated by a periodic gate configuration schematically shown in Fig. 1. In subsequent studies the effect of induced charge density wave correlations in the quantum wire due to the periodic gating was examined, and the optimal regime where insulating current blockade occurs was determined [12]. Other aspects of one-dimensional (1D) electron transport in the presence of modulated Rashba interactions have also been discussed in the literature [13].

In this paper, building on the Peierls-type mechanism identified in Ref. [11], we show how the interplay between a spatially smooth modulated Rashba SOI and an applied magnetic field along the SOI axis may induce a selective opening of energy gaps, providing for spin-polarized electron conductance in a quantum wire. A detailed analysis, based on explicit band diagonalization, bosonization, and extensive DMRG computations, proves that the resulting half-metal phases are stable against repulsive electron-electron interactions, suggesting that the proposed scheme can be realized in the laboratory.

\section{HALF-METAL PHASES FOR NONINTERACTING ELECTRONS}

To elucidate the physics underlying the magnetically controlled half-metal phases, we set out by explaining how a uniform transverse magnetic field parallel to an SOI axis can generate a spin-selective metal-insulator transition. We then specialize to the case of commensurate magnetization and band filling, where one spin projection electron subsystem is pinned by the interactions while the other remains gapless and as a result the system shows a perfect spin-filtering effect.

Using a tight-binding formalism, with the spin-orbit coupled electrons confined to a single 1D conduction channel, we model the system by the Hamiltonian

$$
\begin{aligned}
H= & -t \sum_{n, \alpha}\left(c_{n, \alpha}^{\dagger} c_{n+1, \alpha}+\text { H.c. }\right)-\mu \sum_{n, \alpha} \rho_{n, \alpha} \\
& -i \sum_{n, \alpha, \beta} \gamma_{R}(n)\left[c_{n, \alpha}^{\dagger} \sigma_{\alpha \beta}^{y} c_{n+1, \beta}+\text { H.c. }\right] \\
& +\frac{h_{y}}{2} \sum_{n, \alpha, \beta} c_{n, \alpha}^{\dagger} \sigma_{\alpha \beta}^{y} c_{n, \beta},
\end{aligned}
$$

for now ignoring the electron-electron interaction. Here $c_{n, \alpha}^{\dagger}$ $\left(c_{n, \alpha}\right)$ is the creation (annihilation) operator for an electron with spin $\alpha=\uparrow, \downarrow$ on site $n, \rho_{n, \alpha}=c_{n, \alpha}^{\dagger} c_{n, \alpha}, \quad t$ is the electron hopping amplitude, $\mu$ a chemical potential, $h_{y}$ is the external magnetic field along the SOI axis $\sim \hat{y}$. In the second line of Eq. (1) we assume [11] that the $\hat{y}$ propagating electron modes are confined to their ground state, and that the remaining Rashba SOI modulation can be modeled by $\gamma_{R}(n)=\gamma_{0}+\gamma_{1} \cos (Q n)$, with $\gamma_{0}\left(\gamma_{1}\right)$ being the amplitude 


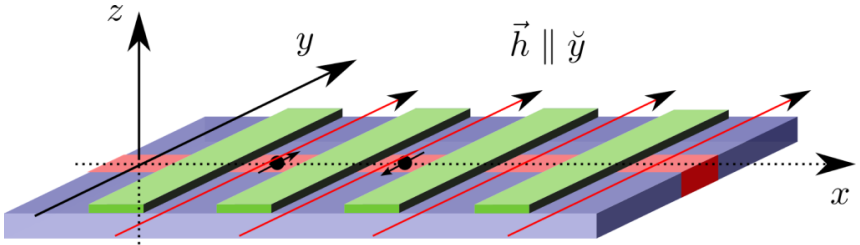

$V_{G} \neq 0$

FIG. 1. A qualitative sketch of the quantum device discussed in this paper. A quantum wire supporting Rashba SOI is gated by a periodic sequence of equally charged top gates. A transverse uniform magnetic field is applied in the direction perpendicular to the current (wire) and the external electric field.

of its uniform (modulated) part. This minimal assumption smooths the unreal sharp interfaces suggested by Fig. 1 and allows for a well-controlled analysis, also in the presence of electron interactions. For transparency and ease of notation we have omitted the modulation of the chemical potential term caused by the modulated gate potential. It can be shown to result only in a band gap renormalization, an effect that can easily be included a posteriori by following a prescription in Ref. [12].

Choosing $\hat{y}$ as spin quantization axis, the uniform part of the $\mathrm{SOI}$ in (1) is seen to split the dispersion relations of the rotated spins by a momentum $\tau q_{0}$, with $q_{0}=\arctan \left(\gamma_{0} / t\right)$ and with $\tau= \pm$ the spin projections along $\hat{y}$. In addition, the $\tau= \pm$ bands are split also by a Zeeman energy $\Delta \varepsilon=-\tau h_{y} / 2$ due to the magnetic field $h_{y}$. For a given filling fraction $v$ and magnetization $m$, the right and left Fermi momenta for each band are given by

$$
k_{F, \tau}^{R / L}=\tau q_{0} \pm k_{F, \tau}^{0},
$$

where $k_{F, \tau}^{0}=(v+\tau m) \pi / 2$. The corresponding values of magnetic field and chemical potential can be obtained from the conditions

$$
\begin{array}{r}
\epsilon_{+}^{0}\left(q_{0} \pm k_{F,+}^{0}\right)-\mu-h_{y} / 2=\epsilon_{-}^{0}\left(-q_{0} \pm k_{F,-}^{0}\right) \\
-\mu+h_{y} / 2=0 .
\end{array}
$$

To assess the impact of the modulated part of the SOI in (1), it is convenient to use a bosonization approach [14]. This will also be practical when we later analyze the role of electronelectron interactions, with bosonization offering an expedient view on correlation effects in the presence of a Rashba SOI [15]. Introducing Bose fields $\varphi_{\tau}$ and their duals $\vartheta_{\tau}$, standard bosonization maps the low-energy sector of the Hamiltonian in (1) to an effective continuum theory $H^{\text {bos }}=H_{0}^{\text {bos }}+H_{\gamma_{1}}^{\text {bos }}$, where

$$
\begin{gathered}
H_{0}^{\mathrm{bos}}=\frac{v_{F, \tau}}{2} \sum_{\tau= \pm} \int d x\left[\left(\partial_{x} \varphi_{\tau}\right)^{2}+\left(\partial_{x} \vartheta_{\tau}\right)^{2}\right] \\
H_{\gamma_{1}}^{\text {bos }}=-\frac{M_{R}}{\pi a_{0}} \sum_{\tau= \pm} \sum_{j= \pm 1} \int d x \sin \left[\left(2 k_{F, \tau}^{0}+j Q\right) x+k_{F, \tau}^{0}\right. \\
\left.+\sqrt{4 \pi} \varphi_{\tau}(x)\right] .
\end{gathered}
$$

Here $v_{F, \tau}=2 \sqrt{t^{2}+\gamma_{0}^{2}} \sin \left(k_{F, \tau}^{0}\right)$ are the Fermi velocities, $M_{R}=\gamma_{1} \sin \left(q_{0} a_{0}\right)$ measures the strength of the modulated
Rashba SOI, and $a_{0}$ is a short-distance cutoff conveniently taken as the lattice spacing. Note that the bosonized Hamiltonian can also be decomposed as $H^{\text {bos }}=H_{+}^{\text {bos }}+H_{-}^{\text {bos }}$, with each piece containing only Bose fields with either $\tau=+$ or $\tau=-$, showing that the rotated spins are good quantum numbers.

From Eq. (4) one concludes that the modulated Rashba SOI can have an effect only at commensurate band fillings given by the conditions

$$
2 k_{F, \tau}^{0} \pm Q \approx 0 \bmod 2 \pi,
$$

since for all other cases the sine term in (4) oscillates rapidly and vanishes upon integration. At finite magnetization the commensurability conditions in (5) are different for each spin projection; when the conditions are met for a given polarization, a relevant perturbation (in the sense of the renormalization group [14]) is present, opening a gap to the corresponding electron excitations. To be precise, when only the spin "+" sector satisfies the commensurability condition, $H_{+}^{\text {bos }}$ is a massive sine-Gordon model and becomes gapful with a mass gap $M_{R}$, while $H_{-}^{\text {bos }}$ describes a gapless Gaussian model. Then the + spin electron subsystem is pinned by the commensurability effect in a long-range ordered quantum configuration while the "-" sector remains gapless and disordered. Charge transport in the gapped sector is forbidden while in the gapless one transport is ballistic. Therefore the wire displays a half-metal behavior [16] and acts as a spin filter. Such a half-metal phase, induced by a magnetic field and a modulated gate voltage, might be experimentally realized and controlled by varying the electron chemical potential via a back gate. As the conducting sector could be turned ON/OFF or even changed to the other spin polarization, the proposed device would be properly called a magnetic spin filter.

The results above, obtained for noninteracting electrons, can easily be checked numerically by explicit band diagonalization. In Fig. 2 we illustrate a simple case by plotting the single-particle dispersion relations for Rashba modulation $Q=\pi$, with band filling $v=3 / 4$ and magnetization $m=1 / 4$.

\section{EFFECT OF THE ELECTRON-ELECTRON CORRELATIONS}

To find out whether the half-metal phases identified above can be realized experimentally, it is crucial to analyze the effect of electron-electron correlations. For this purpose, we here model the screened Coulomb interaction between electrons by an on-site Hubbard interaction

$$
H_{U}=U \sum_{n} \rho_{n, \uparrow} \rho_{n, \downarrow},
$$

to be added to the Hamiltonian in (1).

\section{A. Small $U$ : Bosonization analysis}

The bosonized expression of Eq. (6) in the rotated basis (with spin projections \pm along the $\hat{y}$ axis) takes the form

$$
\begin{aligned}
H_{U}^{\mathrm{bos}}= & \frac{U}{\pi} \int d x\left[\partial_{x} \varphi_{+} \partial_{x} \varphi_{-}+\frac{1}{\pi a_{0}^{2}} \sin \left(\sqrt{4 \pi} \varphi_{+}+2 k_{F,+}^{0} x\right)\right. \\
& \left.\times \sin \left(\sqrt{4 \pi} \varphi_{-}+2 k_{F,-}^{0} x\right)\right\} .
\end{aligned}
$$




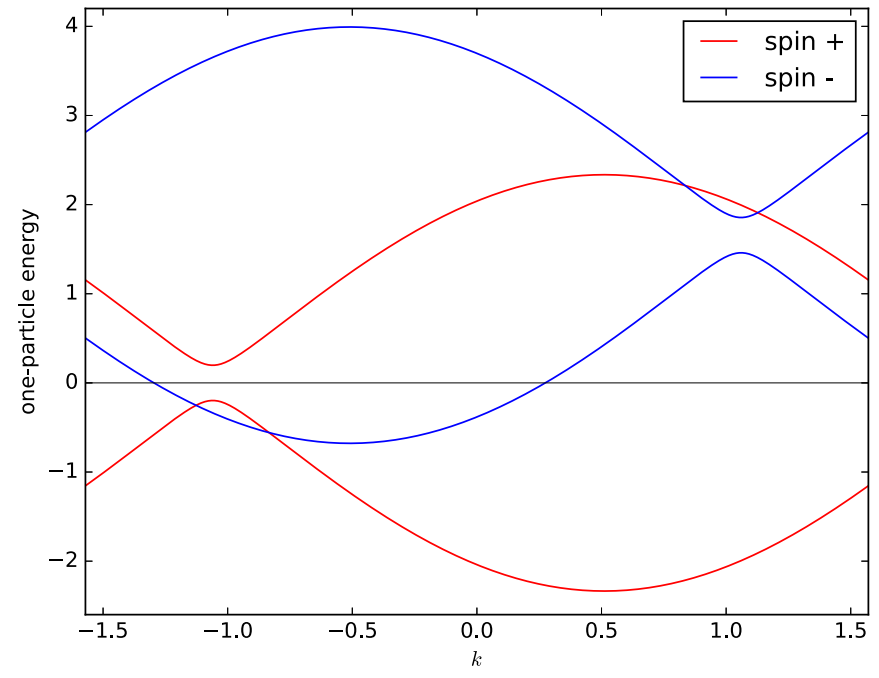

FIG. 2. Single-particle dispersion relation for $Q=\pi, v=3 / 4$, $m=1 / 4$, with Fermi level $\epsilon_{F}=0$. The lower band with spin " + " is completely filled, but the lower band with spin "-" is partially filled. There is a gap to charge excitations with spin + , but no gap to charge excitations with spin - . Rashba coefficients are taken as $\gamma_{0} / t=$ $\tan (\pi / 6)$ and $\gamma_{1} / t=0.2$, filling and magnetization correspond to magnetic field $h_{y} / t=1.78$ and chemical potential $\mu / t=-0.89$.

In a half-metal phase, where condition (5) is satisfied in one spin sector, the sine factors in (7) come out incommensurate, implying that their product averages to zero upon integration. This leaves us with the gradient term in (7), which, however, is exactly marginal and therefore cannot close the gap $\sim M_{R}$. One concludes that electron correlations do not destabilize the magnetically controlled half-metal phases at the low energies where bosonization applies.

\section{B. Larger $U$ : DMRG analysis}

What about intermediate energies where effects from the lattice may play a role? To find out, we have carried out large-scale DMRG computations on the Hamiltonian in (1) with the Hubbard interaction (6) added, $H^{\prime}=H+H_{U}$. The computations were performed in the same rotated spin basis as used above, with electron operators

$$
\left(\begin{array}{c}
d_{n,+} \\
d_{n,-}
\end{array}\right) \equiv \frac{1}{\sqrt{2}}\left(\begin{array}{c}
c_{n, \uparrow}-i c_{n, \downarrow} \\
-i c_{n, \uparrow}+c_{n, \downarrow}
\end{array}\right) .
$$

The Hamiltonian $H^{\prime}$ commutes with the total charge operator $\sum_{n, \tau} d_{n, \tau}^{\dagger} d_{n, \tau}$ and the total spin $y$-component operator $\frac{1}{2} \sum_{n, \tau} \tau d_{n, \tau}^{\dagger} d_{n, \tau}$. As a consequence, the eigenvalues of $\hat{N}_{+}=\sum_{n} d_{n,+}^{\dagger} d_{n,+}$ and $\hat{N}_{-}=\sum_{n} d_{n,-}^{\dagger} d_{n,-}$ are good quantum numbers describing the occupation of states with each spin projection $\tau= \pm$. For a chain of length $L$ with band filling $v$ and magnetization $m$ we then consider the lowest-energy state in the subspace with $N_{+}=L(v+m) / 2$ occupied states with spin + and $N_{-}=L(v-m) / 2$ occupied states with spin - , denoting by $E_{0}\left(N_{+}, N_{-}\right)$the corresponding ground-state energy. One-particle excitation gaps $\Delta_{ \pm}$are defined as the average energy cost of adding or removing an electron with a

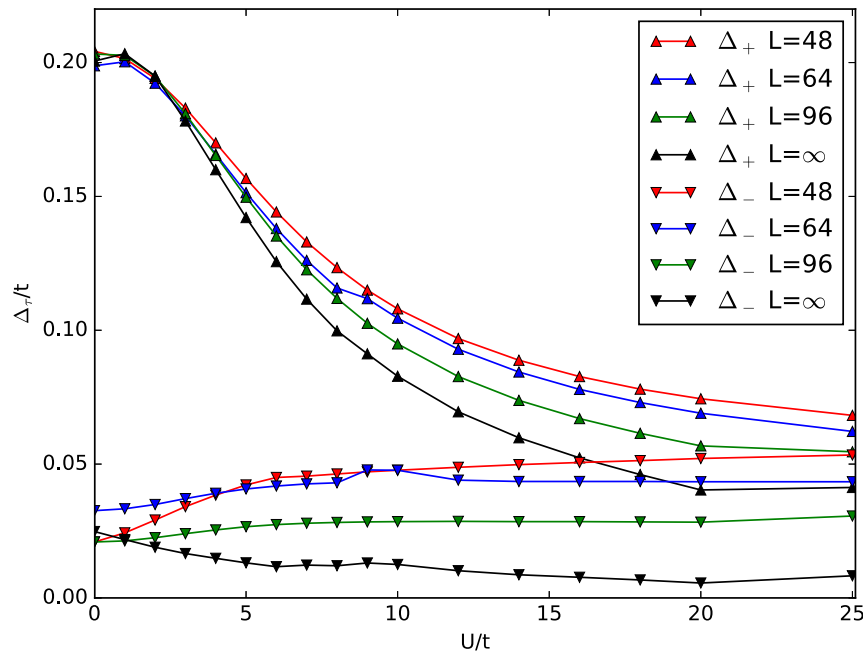

FIG. 3. DMRG results for one-particle gaps as function of $U$ in the half-metal phase depicted in Fig. 2.

given spin projection $\pm[17]$,

$$
2 \Delta_{ \pm}=E_{0}\left(N_{ \pm}+1, N_{\mp}\right)+E_{0}\left(N_{ \pm}-1, N_{\mp}\right)-2 E_{0}\left(N_{+}, N_{-}\right),
$$

and coincide, in the gapped spin sector of a half-metal phase, with the excitation gap $M_{R}$ of the massive sine-Gordon model above. Importantly, this is the gap that determines the current blockade effect in our proposed spin-filter device.

In Fig. 3 we show our DMRG results for the one-particle gaps and their infinite length extrapolation in the half-metal phase sustained by a Rashba SOI modulation $Q=\pi$, filling $v=3 / 4$ and magnetization $m=1 / 4$, with the condition (5) satisfied for spin + , and with the repulsive Hubbard interaction ranging from $U=0$ to $U=25 t$. The remaining Hamiltonian parameters were set to $\gamma_{0} / t=\tan (\pi / 6)$ and $\gamma_{1} / t=0.2$. The computations were carried out for finite-length systems with $L=48,64$, and 96 sites, using the ALPS library [18]. Most of the data points have been obtained keeping 800 states during 30 sweeps. The estimated error for energy measures is $10^{-3} t$, which ensures enough precision for the gaps we report.

In the noninteracting case, as discussed above, the spin + band is half-filled and gapped [with $\Delta_{+}=0.2 t$ in Fig. 3] while the spin - band is quarter-filled and gapless. As seen in Fig. 3, electron-electron repulsion $\sim U$ reduces the gap $\Delta_{+}$, however, without closing it for any $U$. On the contrary, the system seems to stabilize with a different gap in the large $U$ limit. The spin - gap, which vanishes at $U=0$, also scales to zero for any $U$. This last result, however, is highly sensitive to finite-size effects; in particular, the dispersion seen in Fig. 3 at $U=0$ is due to the incommensurability between the band energy minimum of the shifted bands and the discrete finite-length reciprocal lattice.

It is instructive to consider also the two-particle excitation gaps, which describe pure charge or pure spin excitations, related to the bosonic charge and spin fields $\varphi_{c}=\left(\varphi_{+}+\right.$ $\left.\varphi_{-}\right) / \sqrt{2}$ and $\varphi_{s}=\left(\varphi_{+}-\varphi_{-}\right) / \sqrt{2}$, respectively. The charge gap $\Delta_{c}$ is defined by

$$
\begin{aligned}
\Delta_{c}= & \frac{1}{2}\left[E_{0}\left(N_{+}+1, N_{-}+1\right) E_{0}\left(N_{+}-1, N_{-}-1\right)\right. \\
& \left.-2 E_{0}\left(N_{+}, N_{-}\right)\right],
\end{aligned}
$$




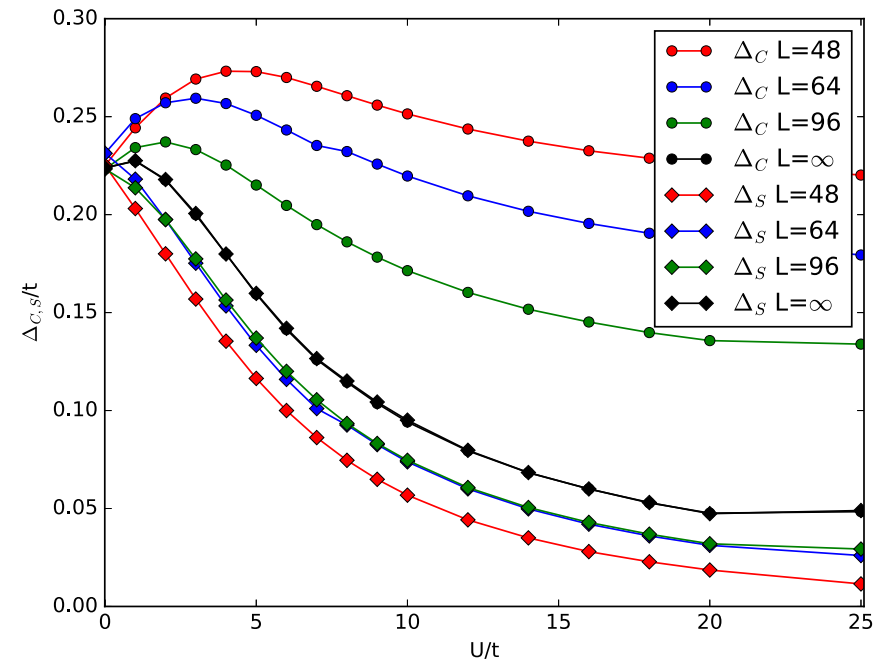

FIG. 4. DMRG results for two-particle gaps as function of $U$ in the half-metal phase depicted in Fig. 2.

while the spin gap $\Delta_{s}$ is defined by

$$
\begin{aligned}
\Delta_{s}= & \frac{1}{2}\left[E_{0}\left(N_{+}+1, N_{-}-1\right)+E_{0}\left(N_{+}-1, N_{-}+1\right)\right. \\
& \left.-2 E_{0}\left(N_{+}, N_{-}\right)\right] .
\end{aligned}
$$

For the noninteracting system $(U=0)$, the two-particle gaps are simply related to the gaps of single particles as $\Delta_{c}=\Delta_{s}=$ $\Delta_{+}+\Delta_{-}$. The presence of electron interactions may change these relations, however. In fact, the more different the charge and spin gaps are, the more correlated the system is, making two-particle gaps sensitive probes of correlation effects.

In Fig. 4 we present the corresponding numerical results for the two-particle gaps. Although the density matrix renormalization (DMRG) data show a marked size dependence, the infinite-length extrapolation following a $1 / L$ law fits remarkably well the finite-size data, showing that the charge and spin gaps remain coincident at any $U$, being the sum of the one-particle gaps. This strongly supports the picture of the system remaining in the same non-correlated phase as when $U=0$. For weak and intermediate electron-electron interactions, with $U \lesssim t$, the only noticeable interaction effect is a small reduction of the single-particle gap (cf. Fig. 3).

\section{PROPOSALS FOR EXPERIMENTAL REALIZATIONS}

Having furnished a proof of concept for a novel type of spin filter device-exploiting the possibility of magnetically controlled half-metal phases in a quantum wire subject to periodic gating — what are the prospects to actually make it work? While an exhaustive analysis goes beyond the scope of this paper, let us attempt a brief appraisal. Given that correlation effects are negligible for the weak to intermediate interaction strength $U / t \lesssim \mathcal{O}(1)$ expected for a gated quantum wire supported by a semiconductor heterostructure [19], the key parameter that determines the functionality of the device is the single-particle gap $M_{R}$, defined above for noninteracting electrons as $M_{R}=2 \gamma_{1} \sin \left(q_{0} a_{0}\right)$. When including the effect from the modulation of the chemical potential due to the periodic gating (cf. Fig. 1), $M_{R}$ gets dressed by the amplitude $\mu_{\text {mod }}$ of the modulation and is replaced by

$$
M_{R, \mu_{\mathrm{mod}}}=\sqrt{M_{R}^{2} \pm \mu_{\mathrm{mod}} M_{R} \cos (\pi v)+\mu_{\mathrm{mod}}^{2} / 4},
$$

with $v$ the band filling, and with the sign $+(-)$ coding for the Rashba and chemical potential modulations being out of phase (in phase) depending on material and design of the setup [12]. As a case study, we use data obtained from an experiment on gate-controlled Rashba interaction in a square asymmetric InAs quantum well [20], assuming that it has been gated to define a single-channel $\mu \mathrm{m}$-range ballistic quantum wire. Combined with data from Ref. [21], we obtain that $\gamma_{1} \approx$ $1 \times 10^{-11} \mathrm{eVm}, q_{0} a_{0} \approx 0.1,4 \mathrm{meV} \leqslant \mu_{\mathrm{mod}} \leqslant 10 \mathrm{meV}$, and $v \approx 0.04$. The value for $\gamma_{1}$ is here obtained from [21] by assuming that it depends on the voltage of the keyboard of top gates (cf. Fig. 1) in the same way as the uniform part $\left(\sim \gamma_{0}\right)$ of the Rashba interaction. This is a reasonable assumption given that the Rashba coupling $\gamma_{1}$ is largely determined by the behavior of the band edge along the growth direction of the heterostructure ( $\sim \hat{z}$ in Fig. 1$)$, implying that its value right below one of the nanosized top gates should be close to that of $\gamma_{0}$ in the case of a single extended top gate. Taking into account random field fluctuations coming from dopant ions is not expected to have a sizable effect on this estimate [12]. With the chemical potential modulation here being out of phase with that of the Rashba SOI [12], Eq. (11) yields the estimate

$$
0.3 \mathrm{meV} \leqslant M_{R, \mu_{\text {mod }}} \leqslant 3.0 \mathrm{meV} \text {. }
$$

To prevent thermal leakage across the single-particle gap that serves to blockade transport of electrons with wrong spin, a device based on the same materials and basic architecture as in Ref. [20] would thus have to operate well below $1 \mathrm{~K}$. Functionality of a device at higher temperatures may be achieved by boosting the effective Rashba couplings [22], or, maybe more workable, by band engineering, using composite materials where the Rashba and chemical potential modulations are in phase instead of out of phase.

\section{CONCLUSIONS}

In summary, in this paper we have shown that a combination of a uniform magnetic field and a gate voltage controlled modulated Rashba SOI may drive a quantum wire into halfmetal phases, with transport only of electrons with a given spin polarization. We have identified the commensurability conditions for the appearance of such phases, and also provided analytical and numerical evidence for their robustness against electron-electron interactions.

Our results hold promise for the design of a magnetic field-controlled spin-filter device, without resorting to injection from ferromagnetic leads. To assess the viability and functionality of such a design requires further work, theoretical as well as experimental.

\section{ACKNOWLEDGMENTS}

This work was partially supported by CONICET (PIP 2015-813) and ANPCyT (PICT 2012-1724), Argentina, the Brazilian Ministry of Education and the $\mathrm{CNPq}$, the Shota Rustaveli National Science Foundation of Georgia through Grant No. FR/265/6-100/14, and by the Swedish Research Council through Grant No. 621-2014-5972. 
[1] S. A. Wolf, D. D. Awschalom, R. A. Buhrman, J. M. Daughton, S. von Molnár, M. L. Roukes, A. Y. Chtchelkanova, and D. M. Treger, Science 294, 1488 (2001).

[2] I. Zutic, J. Fabian, and S. Das Sarma, Rev. Mod. Phys. 76, 323 (2004).

[3] J. Fabian and I. Zutic in Spintronics - From GMR to Quantum Information, edited by S. Blügel, D. Bürgler, M. Morgenstern, C. M. Schneider, and R. Waser (Jülich, Koln, 2009).

[4] D. Bercioux and P. Lucignano, Rep. Prog. Phys. 78, 106001 (2015).

[5] S. Datta and B. Das, Appl. Phys. Lett. 56, 665 (1990).

[6] E. I. Rashba, Sov. Phys. Solid State 2, 1109 (1960); Y. A. Bychkov and E. I. Rashba, J. Phys. C 17, 6039 (1984).

[7] J. Nitta, T. Akazaki, H. Takayanagi, and T. Enoki, Phys. Rev. Lett. 78, 1335 (1997).

[8] Y. Kato, R. C. Myers, A. C. Gossard, and D. D. Awschalom, Nature (London) 427, 50 (2004).

[9] P. Chuang, S.-C. Ho, L. W. Smith, F. Sfigakis, M. Pepper, C.-H. Chen, J.-C. Fan, J. P. Griffiths, I. Farrer, H. E. Beere, G. A. C. Jones, D. A. Ritchie, and T.-M. Chen, Nature Nanotech. 10, 35 (2015).

[10] P. Středa and P. Šeba, Phys. Rev. Lett. 90, 256601 (2003).

[11] G. I. Japaridze, H. Johannesson, and A. Ferraz, Phys. Rev. B 80, 041308(R) (2009).

[12] M. Malard, I. Grusha, G. I. Japaridze, and H. Johannesson, Phys. Rev. B 84, 075466 (2011).

[13] X. F. Wang, Phys. Rev. B 69, 035302 (2004); S. J. Gong and Z. Q. Yang, J. Phys.: Condens. Matter 19, 446209 (2007); F. Mireles and G. Kirczenow, Phys. Rev. B 64, 024426 (2001); X. F. Wang, P. Vasilopoulos, and F. M. Peeters, ibid. 71, 125301 (2005); L. Zhang, P. Brusheim, and H. Q. Xu, ibid. 72, 045347 (2005); L.-G. Wang, K. Chang, and K.-S. Chan, J. Appl. Phys. 99, 043701 (2006); D. Sanchez and L. Serra, Phys. Rev. B 74, 153313 (2006); D. Sanchez, L. Serra, and M.-S. Choi, ibid. 77,
035315 (2008); X. B. Xiao and Y. G. Chen, Europhys. Lett. 90, 47004 (2010).

[14] T. Giamarchi, Quantum Physics in One Dimension (Oxford University Press, Oxford, 2004).

[15] A. V. Moroz, K. V. Samokhin, and C. H. W. Barnes, Phys. Rev. Lett. 84, 4164 (2000); Phys. Rev. B 62, 16900 (2000); M. Governale and U. Zülicke, ibid. 66, 073311 (2002); A. Iucci, ibid. 68, 075107 (2003); Y. Yu, Y. Wen, J. Li, Z. Su, and S. T. Chui, ibid. 69, 153307 (2004); R. G. Pereira and E. Miranda, ibid. 71, 085318 (2005); Y.-X. Li, Y. Guo, and B.-Z. Li, ibid. 72, 075321 (2005); V. Gritsev, G. I. Japaridze, M. Pletyukhov, and D. Baeriswyl, Phys. Rev. Lett. 94, 137207 (2005); F. Cheng and G. Zhou, J. Phys.: Condens. Matter 19, 136215 (2007); J. Sun, S. Gangadharaiah, and O. A. Starykh, Phys. Rev. Lett. 98, 126408 (2007); S. Gangadharaiah, J. Sun, and O. A. Starykh, Phys. Rev. B 78, 054436 (2008); A. Schulz, A. De Martino, P. Ingenhoven, and R. Egger, ibid. 79, 205432 (2009); A. Schulz, A. De Martino, and R. Egger, ibid. 82, 033407 (2010); B. Braunecker, G. I. Japaridze, J. Klinovaja, and D. Loss, ibid. 82, 045127 (2010).

[16] R. A. de Groot, F. M. Mueller, P. G. van Engen, and K. H. J. Buschow, Phys. Rev. Lett. 50, 2024 (1983).

[17] S. R. Manmana, V. Meden, R. M. Noack, and K. Schönhammer, Phys. Rev. B 70, 155115 (2004).

[18] ALPS Collaboration, A. F. Albuquerque et al., J. Magn. Magn. Mater. 310, 1187 (2007); B. Bauer et al., J. Stat. Mech. (2011) P05001.

[19] P. Fulde, Electron Correlations in Molecules and Solids (Springer-Verlag, Berlin, 1991).

[20] D. Grundler, Phys. Rev. Lett. 84, 6074 (2000).

[21] Properties of Lattice-Matched and Strained Indium Gallium Arsenide, edited by P. Bhattacharaya, EMIS Datareviews Series No. 8, IEE (INSPEC, London, 1993).

[22] A. Manchon, H. C. Koo, J. Nitta, S. M. Frolov, and R. A. Duine, Nature Mater. 14, 871 (2015). 